\title{
Susceptibilidad de Leptocybe invasa (Hymenoptera: Eulophidae) a insecticidas en invernadero
}

\author{
Susceptibility of Leptocybe invasa (Hymenoptera: Eulophidae) to \\ insecticides in greenhouse
}

Alfonso Luna-Cruz', Juan M. Vanegas-Rico ${ }^{*}$, Esteban Rodríguez-Leyva ${ }^{3}$ y J. Refugio Lomeli-Flores ${ }^{3}$

\begin{abstract}
1. Universidad Michoacana de San Nicolás de Hidalgo. Instituto de Investigaciones Químico Biológicas. Morelia, Michoacán, México. alunacr(aconacyt.mx
\end{abstract}

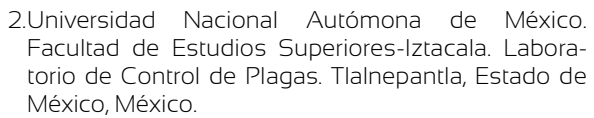
torio de Control de Plagas. Tlalnepantla, Estado de México, México

\author{
3.Colegio de Postgraduados. Programa de \\ Fitosanidad. Texcoco, Estado de México, México. \\ * Autor de correspondencia. \\ entomologo.mexicano@gmail.com
}

\section{ResUmen}

La avispa agalladora del eucalipto, Leptocybe invasa, es una plaga de origen australiano que afecta a plantaciones de Eucalyptus spp. La especie Eucalyptus camaldulensis es el más susceptible hospedero y es ampliamente utilizado en el mundo para madera aserrada, pulpa y carbón; inclusive, es parte del arbolado urbano, como ocurre en varias ciudades de México. La naturaleza exótica de L. invasa y su desarrollo en agallas, dificulta su regulación natural por entomófagos nativos en este país, lo que representa una amenaza para las regiones productoras de eucaliptos y áreas urbanas. El presente estudio evaluó la eficiencia de insecticidas en condiciones de invernadero, mediante aplicación al suelo y foliar, sobre los estados inmaduros de la avispa: huevo, larva joven, larva madura y pupa, utilizando plantas de E. camaldulensis menores de un año. La aplicación de Carbofurán o Imidacloprid en suelo ocasionó mayor mortalidad en huevo (35\% a 79\%) y larva joven (40\% a 75\%), mientras que en larva madura y pupa causó una mortalidad baja $(<5 \%)$. La aplicación de Spirotetramat sobresalió en las aplicaciones foliares, pero no es suficiente por sí sola para controlar a la plaga (mortalidad $<40 \%)$. Con base en los resultados de esta investigación y los antecedentes de la literatura especializada, se sugiere que el manejo químico sobre L. invasa en invernaderos es factible pero solamente como medida emergente. Esto mediante la utilización de Carbofurán o Imidacloprid en aplicación al suelo alternado con una aplicación foliar posterior de Spirotetramat.

PALABRAS CLAVE: agalla, avispa agalladora del eucalipto, eucalipto, plaguicidas, sanidad forestal.

\section{ABSTRACT}

The eucalyptus gall wasp Leptocybe invasa, is an Australian native pest that affects plantations of Eucalyptus spp. E. camaldulensis is the most susceptible host; worldwide it is used for sawn wood and pulp or used as urban tree in many Mexican cities. The exotic nature of $L$. invasa and its development in galls, impedes its natural regulation for native entomophagous in Mexico, which represents a threat for the producing regions of eucalyptus and urban areas. Thus, the present study evaluated the efficiency of insecticides in greenhouse conditions, by means of soil and foliar applications, on the immature stages: egg, young larva, mature larva and pupa, using plants of E. camaldulensis less than one year old. Major mortality was registered in egg (35\% to 79\%) and young larva (40\% to $75 \%$ ) when Carbofuran or Imidacloprid was applied to the soil. The efficiency of these products diminished (mortality $<5 \%$ ) in the stages of mature larva and pupa. The application of Spirotetramat stood out in the application on leaves, but it is not enough on its own to control the plague (mortality $<40 \%$ ). Based on the results of this investigation, and previous studies published in the specialized literature, it is suggested that chemical management on L. invasa in greenhouses is feasible but only as an emergency measure. This, using Carbofuran or Imidacloprid in application to the soil, alternating with an application on the leaves after Spirotetramat.

KEYWORDS: gall, blue gum chalcid, eucalyptus, pesticides, forest health. 


\section{INTRODUCCIÓN}

La avispa Leptocybe invasa, conocida como "blue gum chalcid", "eucalyptus gall wasp" o "avispa agalladora del eucalipto" Fisher \& La Salle (Hymenoptera: Eulophidae), es una plaga que afecta a diferentes especies de Eucalyptus (Myrtales: Myrtaceae) (Mendel, Protasov, Fisher y La Salle, 2004) y a una especie del género Corymbia (Thu, Dell y Burgess, 2009). El ataque de este insecto es más frecuente sobre brotes y follaje joven, ocasionando malformaciones (agallas) en ramas, vena central y peciolo de las hojas (Mendel et al., 2004; Kim, Mendel, Protasov, Blumberg y La Salle, 2008), lo cual puede dañar severamente al arbolado menor de cinco años (Nyeko, 2005), reduciendo su vitalidad y causando disminución de crecimiento. Dichas alteraciones pueden demeritar la calidad de la madera (dos Santos et al., 2018a), causar la pérdida de ramas e incluso la muerte de la planta (Mendel et al., 2004).

Leptocybe invasa es originaria de Australia (Mendel et al., 2004), pero en las últimas dos décadas incrementó su distribución hacia regiones templadas y tropicales con plantaciones de eucaliptos. Su primer registro y descripción como nueva especie ocurrió en Israel (Mendel et al., 2004); posteriormente, amplió su distribución a otros países del mundo (European and Mediterranean Plant Protection Organization [Emppo], 2006), reportándose en África:Algeria, Kenya, Morocco, Uganda (Nesser, Prinsloo y Neser , 2007; Nyeko, Mutitu, Otieno, Ngae y Day, 2010), Asia: India (Jacob, Devaraj y Natarajan, 2007), Vietnam (Thu et al., 2009), China (Tang, Wang, Wan, Ren y Peng, 2008) y América: Brasil (Wilcken y Filho, 2008), Estados Unidos (Wiley y Skelley, 2008), Argentina (Aquino, Botto, Loiácono y Pathauer, 2011), México (Vanegas-Rico, Lomeli-Flores, Rodríguez-Leyva, Jiménez-Quiróz y Pujade-Villar, 2015), Paraguay (Benítez, Costa, de Moraes y Godziewsky, 2014) y Uruguay (Jorge, Martínez, Gómez y Bollazzi, 2016).

Entre los principales hospederos de L. invasa se menciona a Eucalyptus camaldulensis, E. tereticomis y E. urophylla (Zheng et al., 2014), especies de importancia forestal por la obtención de pulpa y madera sólida en distintas regiones del mundo (Mendel et al., 2004; Zheng et al., 2014; Rejmánek y Richardson, 2011; Tong, Ding, Zhang, Yang y Huang, 2016). Los registros sobre el impacto económico de L. invasa incluyen la cancelación de producción de E. camaldulensis en Israel (Mendel et al., 2004), la afectación de 200 ha en Maranhão, Brasil (dos Santos et al., 2018a), 20000 ha en Tanzania (Petro e Iddi, 2017) y como caso extremo, se estimaron $2.5 \times 10^{6}$ ha afectadas en India (Kulkarni, 2010).

En México, L. invasa se reportó inicialmente en la Ciudad de México, Estado de México, Jalisco y Tamaulipas, desarrollándose sobre E. camaldulensis de áreas urbanas (Vanegas-Rico et al. 2015). Después de la alerta federal (Comisión Nacional Forestal [Conafor], 2015), se registró en otros estados del norte y región central, ninguno de ellos con grandes extensiones de plantaciones, ya que las regiones productoras de eucaliptos de México se ubican predominantemente en el sureste (Conafor, comunicación personal). Hasta el momento, únicamente en Tamaulipas (región noreste del país), se registró la infestación de $L$. invasa en plantaciones jóvenes de E. camaldulensis, con una incidencia de $70 \%$ y una severidad de hasta $80 \%$ de la cobertura vegetal (Conafor, comunicación personal).

La remoción o poda selectiva, es la primera acción de control cuando la infestación se detecta en árboles jóvenes (Senthilkumar, Murugesan y Thangapandian, 2013; Zheng et al., 2014). En plantaciones forestales se seleccionan clones resistentes (Zhang, Kang, Chen, Tang y Lin, 2012) y se evalúan insecticidas sistémicos, de contacto, o la combinación de ambos, para tratamiento preventivo en semilla, plántulas y esquejes (Jhala, Patel y Vaghela, 2010, Aregowda, Prabhu y Patil, 2010). Bajo este esquema preventivo y considerando la susceptibilidad de las plantas jóvenes (Thu et al., 2009), se deben buscar estrategias de manejo.

\section{OBJETIVOS}

Evaluar el efecto de insecticidas químicos sobre diferentes estados desarrollo de Leptocybe invasa en plantas de eucalipto jóvenes en condiciones de invernadero, como una medida emergente de control. 


\section{MATERIALES Y MÉTODOS}

\section{Plantas}

La investigación se realizó en las instalaciones del Colegio de Postgraduados, Texcoco, México; en condiciones de invernadero $\left(22^{\circ} \mathrm{C} \pm 8{ }^{\circ} \mathrm{C}, 70 \%-80 \% \mathrm{HR}\right)$. Se utilizaron plantas de E. camaldulensis de nueve meses de edad, adquiridas en un vivero comercial de Cuautla, Morelos, México. Los árboles estaban sembrados en bolsas de polietileno (5 L) con suelo agrícola de esa región y libre de aplicación de insecticidas. Las plantas se mantuvieron en las mismas bolsas durante el desarrollo de los experimentos, el agua se proporcionó con un sistema de riego por goteo y se incluyó nutrición universal (Steiner, 1961), con ligeras modificaciones.

\section{Insectos}

Los insectos se obtuvieron de ramas de eucaliptos infestadas, procedentes del noreste de la Ciudad de México. Este material se mantuvo en bolsas herméticas con papel absorbente (Vanegas-Rico et al., 2015) para su trasporte al laboratorio de control biológico del Colegio de Postgraduados. Las ramas se mantuvieron en agua a temperatura ambiente en recipientes plásticos de $3 \mathrm{~L}$ para esperar la emergencia de las avispas. Las cuales se alimentaron con miel de abeja y se trasportaron en viales eppendorf para infestar las plantas de eucalipto, se colocaron en bolsas elaboradas con organza $(25 \mathrm{~cm} \times 20$ $\mathrm{cm})$ sobre las ramas. La bolsa de organza se sujetó a la rama de tal forma que las avispas no escaparan y ovipositarán en las hojas de eucalipto. Este procedimiento se repitió semanalmente durante 80 días para tener agallas de diferente tiempo de desarrollo y asemejar las condiciones de infestación natural.

\section{Insecticidas}

La investigación se desarrolló con dos experimentos, a) aspersión foliar y b) aplicación en suelo, utilizando insecticidas de diferente grupo toxicológico; en ambos, casos solo se realizó una aplicación (Tabla 1). Cada tratamiento se integró con 10 plantas, incluyendo un testigo con agua destilada, en todos los tratamientos se utilizó el penetrante Inex-A (2.0 $\mathrm{mL} \mathrm{L}^{-1}$ de agua). Las aplicaciones de insecticidas se realizaron después de la liberación y establecimiento de L. invasa.

\section{Experimento 1. Aspersión foliar}

El experimento evaluó tres insecticidas de acción sistémica: Imidacloprid (0.12 g i. a. $\mathrm{L}^{-1}$ de agua), Spirotetramat (0.34 g i. a. $L^{-1}$ de agua) y Dimetoato ( 0.6 g i. a. $L^{-1}$ de agua); además de un insecticida de efecto traslaminar: Abamectina (0.036 g i. a. $\mathrm{L}^{-1}$ de agua). Los árboles se colocaron fuera del invernadero y se les aplicó el insecticida correspondiente, con una bomba motorizada (Aritmisu®, Osaka, Japón) a una presión de $1.379 \mathrm{mPa}$ (200 PSI), sobre el haz y envés de las hojas hasta el punto de escurrimiento. Después de la aplicación se dejaron secar a temperatura ambiente por 20 minutos y se introdujeron al invernadero, distribuyéndolas completamente al azar. Cada tratamiento contó con 5 réplicas, en dos repeticiones en el tiempo, es decir, 10 réplicas en total; asumiendo una planta de eucalipto como unidad experimental.

\section{Experimento 2. Aplicación de insecticidas sisté- micos al suelo}

El experimento tuvo cinco tratamientos, los cuales consistieron en dos concentraciones de Imidacloprid ( $4.0 \mathrm{y}$ 2.1 g i. a.), dos de Carbofurán (0.5 y 0.75 g i. a.) y un testigo. El Imidacloprid se diluyó en agua destilada, se agregó el penetrante Inex-A (2.0 $\mathrm{mL} \mathrm{L}^{-1}$ de agua), posteriormente se aplicaron $500 \mathrm{~mL}$ de solución en cada planta de eucalipto. El Carbofurán granulado se distribuyó en tres orificios (1 $\mathrm{cm}$ de profundidad) circundantes al tallo, cubriéndose con el sustrato de la maceta y luego se regó con agua corriente. Cada tratamiento contó con 10 réplicas, en dos repeticiones en el tiempo, es decir, 20 réplicas en total; asumiendo una planta de eucalipto como unidad experimental. 
TABLA 1. Insecticidas evaluados contra Leptocybe invasa en condiciones de invernadero.

\begin{tabular}{cccc}
\hline Ingrediente activo & Insecticida & Grupo químico & Concentración* \\
\hline Carbofuran & Lucarfuram 5G & Carbamatos & 1.25 \\
Imidacloprid & Confidor 350 SC & Neonicotinoides & 0.40 \\
Abamectina & Agrimec 1.8\% CE & Avermectina & 0.20 \\
Dimetoato & Dimetri 400 & Organofosforado & 0.92 \\
Spirotetramat & Movento 150 OD & Ácidos tetrámicos & 0.32 \\
\hline
\end{tabular}

* Concentración: gramos de ingrediente activo por litro de producto o agua

\section{Evaluación}

La eficiencia de los insecticidas se registró después de siete días de la aplicación de insecticidas y se repitió semanalmente, con una duración de 21 días y 35 días en el primer y segundo experimento, respectivamente. Las evaluaciones consistieron en cortar aleatoriamente cinco hojas por planta con presencia de agallas, las cuales se guardaron en bolsas herméticas y fueron trasportadas al laboratorio. Las agallas se revisaron mediante cortes longitudinales para contabilizar los organismos vivos o muertos de cada estado de desarrollo de L. invasa. La determinación de dichos estados de desarrollo se estableció con base en los cambios físicos de la agalla (Mendel et al., 2004), considerando además el tiempo aproximado después de la oviposición: huevo ( 3 días a 8 días), el tejido vegetal presenta orificios de oviposición a través de los cuales se producen secreciones resinosas durante los primeros días; larva joven (28 días a 35 días), cada una se desarrolla individualmente dentro de una cavidad, el tejido presenta abultamiento y una coloración exterior verde que la mayoría de las ocasiones denota brillo; larva madura (45 días a 55 días), el organismo ocupa todo el espacio de la cavidad donde se desarrolla, el tejido vegetal tiene usualmente una coloración exterior rosa-rojiza con pérdida de brillo; pupa (55 días a 70 días), el organismo es blanquecino y presenta las características típicas de una pupa exarata, es decir, se aprecia la división del cuerpo en cabeza, tórax y abdomen, la coloración de la agalla usualmente es rojiza, aunque en algunas ocasiones su coloración y tamaño solo difieren de agallas más jóvenes por la falta de brillo. El criterio de mortalidad consistió en la falta de turgencia o en la coloración oscura en el tejido de los estados inmaduros, la cual es de color blanquecina cuando los organismos presentan buena salud.

\section{Análisis estadístico}

La proporción de organismos muertos (No. organismos muertos recolectados en cinco hojas/No. de organismos totales recolectados en cinco hojas) de cada estado de desarrollo de L. invasa se comparó entre tratamientos en cada semana de evaluación mediante una ANOVA, realizando una separación de medias de Tukey $(\alpha=0.05)$ con el programa SPSS v. 22 (Macintosh, USA). Esto previo a la comprobación de los supuestos de normalidad y homogeneidad de varianzas.

\section{Resultados}

\section{Aspersión foliar}

En la primera semana de evaluación no se registró mortalidad en ningún estado de desarrollo de L. invasa, mientras que en la segunda semana los valores fueron inferiores a $5 \%$ o bien no se registró mortalidad. $\left(\mathrm{F}_{4,49}=1.26 ; \mathrm{P}<0.001\right)$, contrastando el Dimetoato como el insecticida con menor mortalidad sobre L. invasa (intervalo de $0.5 \%$ a $2 \%$ ) y el Spirotetramat como el más eficiente sobre todos los estados de desarrollo evaluados, aunque los valores de mortalidad fueron inferiores a $40 \%$. El insecticida Imidacloprid causó mortalidad intermedia, con valores de $0 \%$ a $25 \%$ en todos los estados de desarrollo (Fig. 1). 


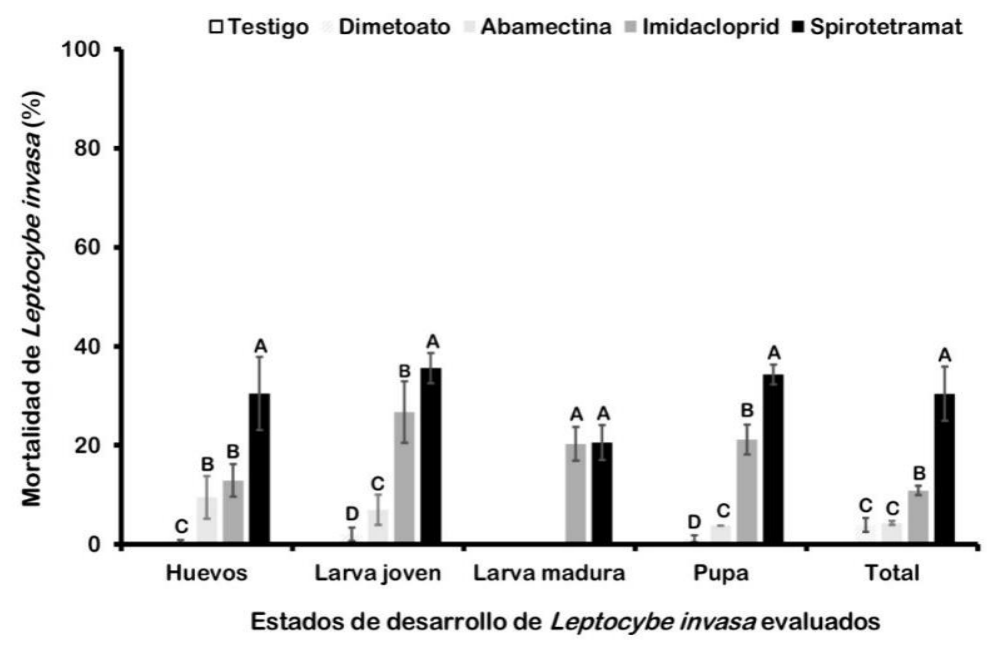

FIGURA 1. Mortalidad de Leptocybe invasa con diferentes insecticidas, 21 días posteriores a la aplicación foliar.

\section{Aplicación de insecticidas sistémicos al suelo}

El huevo de L. invasa fue el estado de desarrollo donde se observó la mayor mortalidad (30\%) en la primera semana de evaluación, posteriormente aumentó con todos los insecticidas, registrando la mayor mortalidad ( $81 \%$ a $94 \%$ ) 28 días después de la aplicación y disminuyó la siguiente semana $(65 \%$ a $83 \%)$. En general, durante las tres primeras semanas, el tratamiento que presentó mayor mortalidad fue el insecticida Carbofurán con la dosis de $0.75 \mathrm{~g}$ i. a., aunque en la última evaluación (35 días), Imidacloprid ( $2.1 \mathrm{~g}$ i. a.) alcanzó 83\% de mortalidad de huevo, siendo el valor más alto $\left(\mathrm{F}_{4,75}=10.58, \mathrm{P}<0.001\right)$.

La mortalidad de larvas jóvenes fue menor a 30\% durante la primera semana en todos los tratamientos y aumentó en las siguientes evaluaciones, registrando la mayor mortalidad 28 días después de la aplicación (intervalo de $58 \%$ a $79 \%$ de mortalidad). Con excepción de la primera semana posterior a la aplicación de los insecticidas, a partir de 14 días $\left(F_{4,92}=2.64, P=0.058\right)$, no hubo diferencia en la mortalidad ocasionada por los insecticidas Imidacloprid y Carbofurán en las concentraciones más altas $(2.1$ y $0.75 \mathrm{~g}$ i. a., respectivamente) (Fig. 2).

En larva madura se registraron mortalidades menores a $20 \%$ en todas las evaluaciones. Los valores más altos ocurrieron en el periodo de 28 días a 35 días después de la aplicación (intervalo $0.7 \%$ a 18\%), sobresaliendo ligeramente el insecticida Imidacloprid en su dosis baja (1.4 g de i. a.). El resto de los tratamientos presentaron mortalidades cercanas a $10 \%$ (Fig. 2).

El estado de pupa de las avispas no se afectó por los insecticidas, la máxima mortalidad fue cercana a $10 \%$ y se registró con Carbofurán en su concentración alta, a los 35 días posteriores a la aplicación.

\section{DISCUSIÓN}

El desarrollo de agallas es una estrategia evolutiva de algunos insectos que les proporciona refugio contra factores abióticos y al mismo tiempo facilita la obtención de alimento para sus crías (Price, Fernandes y Waring, 1987; Stone y Schönrogge, 2003). La irrupción de especies galícolas como L. invasa hacia sistemas de explotación forestal puede ocasionar grandes pérdidas (dos Santos et al., 2018a) y, por ello, se recurre al uso de insecticidas como primera medida de control (Aquino et al., 2011). La eficiencia de los insecticidas químicos contra este tipo de organismos está limitada a la capacidad de penetración del producto a través de los tejidos vegetales o bien circulación sistémica de estos. Es posible que la doble sistemicidad favoreciera la acción de Spirotetramat (Brück et al., 2008) en 
la mortalidad de inmaduros de L. invasa. Este tipo de aplicación es más efectivo cuando las poblaciones de insectos galícolas son estacionales, por lo que el calendario de aplicaciones se ajusta con las primeras emergencias de los adultos (Eliason y Potter, 2001) los cuales están más expuestos. Esto se dificulta en el caso de L. invasa, porque sus poblaciones suelen traslaparse sobre el mismo árbol, existiendo frecuentes emergencias de adultos durante largos periodos del año en varios países (Mendel et al., 2004; Tang et al., 2008; Jorge et al., 2016), incluida la ciudad de México (Vanegas-Rico, datos sin publicar), lo cual dificulta aún más su control.

Respecto a la aplicación de insecticidas sistémicos en suelo, Carbofurán e Imidacloprid fueron los que ocasionaron mayor mortalidad en la etapa de huevo y larva joven de L. invasa. Esta eficiencia disminuyó a través del tiempo y conforme aumentó el desarrollo del insecto. Es probable que existiera mayor contacto de los químicos sobre el estado de huevo, debido a que el tejido vegetal aún no se desarrolla para proteger a los organismos. Este efecto insecticida al parecer continúa en los primeros días después de la formación de la larva. Durante el desarrollo larval, $L$. invasa se alimenta de un tejido verde que se forma en toda la pared interna, en la cual las células acumulan proteínas y lípidos que proporcionan alimento para las larvas, mientras que el exterior tiene una capa blanquecina semirrígida que parece actuar como barrera de protección (dos Santos et al., 2018b).

Los resultados de los insecticidas evaluados en nuestro estudio sugieren que Carbofuran en ambas dosis $(0.5$ y 0.75 g de i.a), causa $\geq 75 \%$ de mortalidad en huevo y larva joven; mientras que la aplicación foliar demostró cierto control sobre larvas maduras y pupas. La combinación de ambas formas de aplicación, o bien la combinación de insecticidas de diferentes grupos toxicológicos, debería mejorar el control de la plaga. Esto se basa en las investigaciones de otros autores, quienes recomiendan utilizar la combinación de Carbofurán 3G (1 g por planta) e Imidacloprid $(0.25 \mathrm{~mL}$ $\left.\mathrm{L}^{-1}\right)$ para reducir cerca de $50 \%$ de agallas en plantas jóvenes (Aregowda et al., 2010). Otros autores sugieren usar primero Carbofurán 3G (4 g por planta) y una segunda aplicación a base de Imidacloprid $\left(0.4 \mathrm{~mL} \mathrm{~L}^{-1}\right)$ (Chakrabarti, 2015), o bien aplicar Dimetoato ( $\left.2 \mathrm{~mL} \mathrm{~L}^{-1}\right)$ o Imidacloprid (1 $\left.\mathrm{mL} \mathrm{L}^{-1}\right)$ cada 15 días (Senthilkumar, 2013). En el presente trabajo únicamente se consideró una aplicación por los cotos que podría representar.
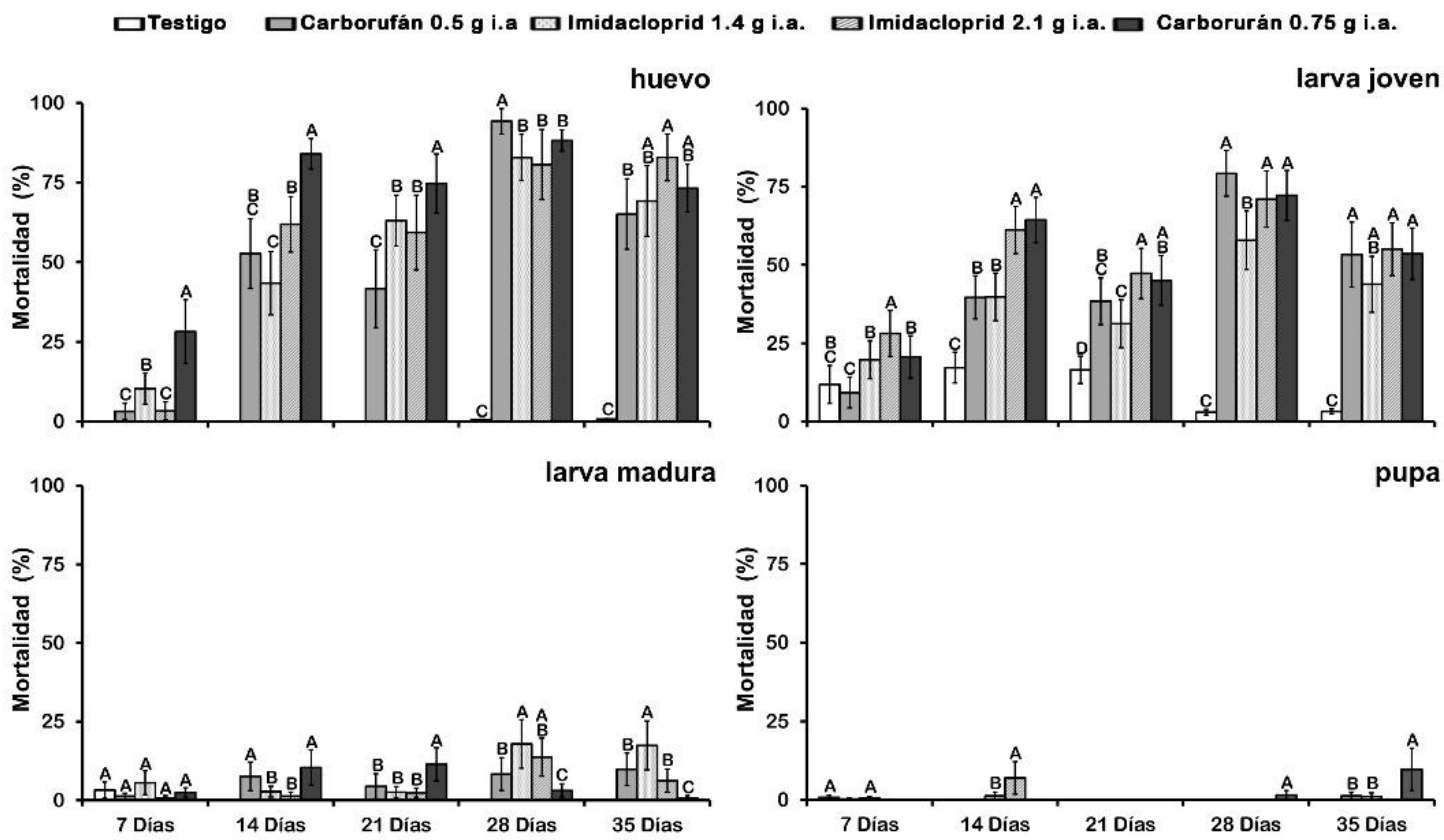

FIGURA 2. Mortalidad de distintos estados de Leptocybe invasa durante 35 días, con insecticidas sistémicos aplicados al suelo. 
La clasificación propuesta en este trabajo para las larvas (joven y madura), aunque no contempla instar específico, proporcionó información relevante sobre dos aspectos: a) el periodo de mayor susceptibilidad de la plaga ocurre aproximadamente un mes después de la oviposición. El tejido de los eucaliptos recién dañados por el ovipositor de L. invasa presenta secreciones hialinas, su detección puede facilitar la planeación de una medida preventiva ya que se ha documentado que la incidencia e infestación puede alcanzar en pocos meses valores cercanos o superiores a 75\% (Roychoudhury, Chandra y Singh, 2016); b) la baja mortalidad de las larvas maduras (después de mes y medio de la oviposición) y pupa, serviría como referencia para considerar la integración de control biológico, ya que los parasitoides evaluados contra $L$. invasa prefieren el estado de pupa o muy cercano a pupar (Protasov, Doganlar, La Salle y Mendel, 2008). Esto es un tema de oportunidad, ya que hasta el momento se carece de estudios sobre compatibilidad de dichas estrategias.

El primer programa de control biológico clásico de esta plaga se inició en Israel hace 10 años (Kim et al., 2008) y se califica como exitoso actualmente se ha desarrollado en países como India (Shylesha, 2008), Sudáfrica (DittrichSchröder et al., 2014, Luo, Arnold, Lu y Lin 2014) y Brasil (Masson et al., 2017). Mientras que, en México, la estrategia actual se basa en un sistema de alerta y monitoreo frecuente (Vanegas-Rico et al., 2015). En arbolado urbano se practica la remoción mecánica de ramas o del árbol completo, además de evitar el desplazamiento de material de poda hacia lugares donde aún no se registra la presencia de $L$. invasa. En la Ciudad de México se inició un programa de monitoreo mediante trampas amarillas y se desarrollan estudios espaciotemporales en algunos parques, además de la búsqueda de parasitoides nativos (Vanegas-Rico datos sin publicar). La experiencia en otras regiones del mundo enfatiza que un enfoque unitario con manejo químico es difícil de aplicar en grandes extensiones naturales o reforestadas, por lo que se considera incosteable y perjudicial para el ambiente (Dittrich-Schröder, 2014, Zheng et al., 2016) y, posiblemente, solo sirva como práctica emergente en invernaderos (Jhala et al., 2010; Ramos, 2015).
En condiciones de invernadero es más factible mantener un control químico, complementado con la eliminación de plantas que presenten agallas; previo a la salida del vivero, para evitar la dispersión de la plaga. Por otro lado, la aplicación de insecticidas al suelo es una alternativa compleja en viveros comerciales donde las plantas se producen en tubetes o bandejas.

\section{CONCLUSIONES}

La aplicación foliar con Spirotetramat afectó a todos los estados de desarrollo de L. invasa, produciendo una mortalidad inferior a 40\%. La aplicación al suelo de Carbofuran o de Imidacloprid afectó a los estados de desarrollo de huevo y larva joven, observándose la mayor eficiencia 35 días después de la aplicación (mortalidad $\approx$ $75 \%$ ). La presente investigación sugiere que el uso de estos insecticidas representa una medida de emergencia a corto plazo en viveros que tengan estados iniciales (huevo y larva joven) de L. invasa. No se descarta su compatibilidad potencial dentro de un manejo integrado que tenga como base el control biológico con parasitoides; los cuales prefieren larvas maduras o pupas para desarrollarse. Por ello, sería conveniente realizar estudios de toxicidad de insecticidas sobre agentes de control biológico para lograr una eventual compatibilidad de los dos sistemas de manejo.

\section{RECONOCIMIENTOS}

Se agradece a Conafor por el apoyo en el proyecto PM154029 "Acciones tendientes al manejo integrado de la avispa agalladora del eucalipto Leptocybe invasa en el distrito federaly en los municipios circunvecinos del estado de México". Igualmente se agradece a la Lic. Lorna Angélica López Nicolás (Directora), al Biol. Gustavo Vera-Ramírez y al resto del personal del Bosque de San Juan de Aragón por su apoyo para la obtención de material biológico de L. invasa.

\section{REFERENCIAS}

Aquino, D. A., Botto, E. N., Loiácono, M. S. \& Pathauer, P. (2011). "Avispa de la agalla del eucalipto", Leptocybe invasa Fischer \& Lasalle (Hymenoptera: Eulophidae: Tetrastichinae), en Argentina. Revista de Investigaciones Agropecuarias, 37(2), 159-164. 
Aregowda, J., Prabhu, S. T., \& Patil, R. S. (2010). Evaluation of botanicals and synthetic insecticides against eucalyptus gall wasp, Leptocybe invasa (Eulophidae: Hymenoptera). Karnatak a Journal of Agricultural Sciences, 23(1), 200-202.

Benítez, E. A., Costa, V. A., de Moraes, G. J., \& Godziewsky, D. (2014). First record of Leptocybe invasa Fisher \& La Salle (Hymenoptera: Eulophidae) and Rhombacus eucalypti Ghosh \& Chakrabarti (Acari: Eriophyidae) from Paraguay. Boletin del Museo Nacional de Historia Natural de Paraguay, 18(1), 129-132.

Brück, E., Elbert, A., Fisher, R., Krueger, S., Kühnhold, J., Klueken, A. M., Nauen, R., Niebes, J. F., Reckmann, U., Schnorbach, H. J., Steffens, R., \& Waetermeulen X. V. (2008). Movento ${ }^{\circledR}$, an innovative ambimobile insecticide for sucking insect pest control in agriculture: Biological profile and field performance. Crop Protection, 28(10), 838-844.

Chakrabarti, S. (2015). Studies on efficacy of some insecticidal molecules against blue gum chalcid, Leptocybe invasa Fisher \& La Salle (Hymenoptera: Eulophidae) in Eucalyptus. Current Biotica, 8(4), 428-431.

Comisión Nacional Forestal [Conafor] (2015). Alerta Fitosanitaria. Avispa Agalladora del eucalipto (Leptocybe invasa). Recuperado de http://www.conafor.gob.mx:8080/documentos/docs/15/6201 Leptocybe $\% 20$ invasa.pdf

Dittrich-Schröder, G., Harney, M., Neser, S., Joffe, T., Bush, S., Hurley, B. P., Wingfield, M J., \& Slippers, B. (2014). Biology and host preference of Selitrichodes neseri: A potential biological control agent of the Eucalyptus gall wasp, Leptocybe invasa. Biological Control, 78, 33-41. doi: 10.1016/j.biocontrol.2014.07.004

dos Santos, G. B, Vidaurre, G. B., Câmara, A. P., Valin, M., da Silva Oliveira, J. T., Soliman, E. P., \& Lopez, Y. M. (2018a). Efecto del ataque de Leptocybe invasa en plantaciones de eucalipto destinadas a la producción de pulpa de celulosa kraft. Maderas. Ciencia y Tecnología, 20(2). doi: 10.4067/S0718-221X2018005000014

dos Santos, R. M. I., García, F. B., de Alvarenga, D. R., Rodrígues, B. L., Salminen, J. P., \& Stenbauer, M. J. (2018b). Functional compartmentalisation of nutrients and phenolics in the tissues of galls induced by Leptocybe invasa (Hymenoptera: Eulophidae) on Eucalyptus camaldulensis (Myrtaceae). Astral Entomology, 57(2), 238246. doi: $10.1111 /$ aen.12336

Eliason, E. A. \& Potter, D. A., (2000). Impact of Whole-Canopy and Systemic Insecticidal Treatments on Callirbytis cornigera (Hymenoptera: Cynipidae) and Associated Parasitoids on Pin Oak. Journal of Economic Entomology, 93, 165-171.

European and Mediterranean Plant Protection Organization [Emppo] (2006). Addition of Leptocybe invasa to the alert list. EPPO Reporting service, 9-10. Recuperado de https://archives.eppo.int/EPPOReporting/2006/Rse-0609.pdf

Jacob, J. P., Devaraj, R., \& Natarajan, R. (2007). Outbreak of the invasive gall-inducing wasp Leptocybe invasa on eucalypts in India. Newsletter of the Asia-Pacific Forest Invasive Species Network, 8, 4-5.

Jhala, R. C., Patel, M. G., \& Vaghela, N. M. (2010). Effectiveness of insecticides against blue gum chalcid, Leptocybe invasa Fisher \& La Salle (Hymenoptera: Eulophidae), infesting eucalyptus seedlings in middle Gujarat, India. Karnataka Journal of Agricultural Sciences, 23(1), 84-86.

Jorge, C., Martínez, G., Gómez, D., \& Bollazzi, M. (2016). First record of the eucalypt gall-wasp Leptocybe invasa (Hymenoptera: Eulophidae) from Uruguay. Bosque, 37(3), 631-636. doi: 10.4067/S0717-92002016000300020.

Kim, I. K., Mendel, Z., Protasov, A., Blumberg, D., \& La Salle, J. (2008). Taxonomy, biology, and efficacy of two Australian parasitoids of the eucalyptus gall wasp, Leptocybe invasa Fisher \& La Salle (Hymenoptera: Eulophidae: Tetrastichinae). Zootaxa, 1910, 1-20. doi: $10.5281 /$ zenodo.184546

Kulkarni, H. D. (2010). Screening eucalyptus clones against Leptocybe invasa Fisher and La Salle (Hymenoptera: Eulophidae). Karnataka Journal Agricultura Sciences, 23(1), 87-90.

Lagunes-Tejeda, A. \& Rodríguez-Maciel, J. C. (1992). Grupos toxicológicos de insecticidas y acaricidas: los mecanismos de resistencia como base para el manejo de insecticidas y acaricidas agrícolas. Colegio de Postgraduados, México.

Luo, J., Arnold, R., Lu, W., \& Lin, Y. (2014). Genetic variation in Eucalyptus camaldulensis and E. tereticornis for early growth and susceptibility to the gall wasp Leptocybe invasa in China. Euphytica, 196(3), 397-411. doi: 10.1007/s10681-013-1042-8

Masson, M. V., Tavares, W. D. S., Lopes, F. D. A., Souza, A. R. D., Ferreira-Filho, P. J., Barbosa, L. R., Wilcken C. F., \& Zanuncio, J. C. (2017). Selitrichodes neseri (Hymenoptera: Eulophidae) recovered from Leptocybe invasa (Hymenoptera: Eulophidae) galls after initial release on Eucalyptus (Myrtaceae) in Brazil, and data on its biology. Florida Entomologist, 100(3), 589-593. doi: 10.1653/024.100.0316

Mendel, Z., Protasov, A., Fisher, N., \& La Salle, J. (2004). Taxonomy and biology of Leptocybe invasa gen. \& sp. n. (Hymenoptera: Eulophidae), an invasive gall inducer on Eucalyptus. Australian Journal of Entomology, 43(2), 101-113. doi: 10.1111/j.14406055.2003.00393.x

Nesser, S., Prinsloo, G. L., \& Neser, O. C. (2007). The eucalypt leaf, twig and stem galling wasp, Leptocybe invasa, now in South Africa. Plant Protection News, 72, 1-2. 
Nyeko, P. (2005). The cause, incidence and severity of a new gall damage on Eucalyptus species at Oruchinga refugee settlement in Mbarara district, Uganda. Uganda Journal of Agricultural Sciences, 11, 47-50

Nyeko, P., Mutitu, K. E., Otieno, B. O., Ngae, G. N., \& Day, R. K. (2010). Variations in Leptocybe invasa (Hymenoptera: Eulophidae) population intensity and infestation on eucalyptus germplasms in Uganda and Kenya. International Journal of Pest Management, 56, 137 144. doi: 10.1080/09670870903248835

Petro, R. \& Iddi, S. (2017). Leptocybe invasa and its effects on young plantations of commercial Eucalyptus species in Tanzania. International Journal of Agriculture and Forestry, 7(1), 23-27. doi: 10.5923/j.ijaf.20170701.04

Price, P. W., Fernandes G.W., \& Waring G. L. (1987). Adaptive nature of insect galls. Environmental Entomology, 16(1), 15-24. doi.org/10.1093/ee/16.1.15

Protasov, A., Doganlar, M., La Salle, J. \& Mendel, Z. (2008). Occurrence of two local megastigmus species parasitic on the Eucalyptus gall wasp Leptocybe invasa in Israel and Turkey. Phytoparasitica, 36, 449459.

Ramos, S. O. (2015). Efecto de insecticidas sobre la formación y desarrollo de las agallas de Leptocybe invasa en eucaliptos. Revista FAVE-Ciencias Agrarias, 14, 1-7.

Rejmánek, M. \& Richardson, D. M. (2011). Eucalypts. En D. Simberloff \& M. Rejmánek (Eds), Encyclopedia of biological invasions (pp. 203 209). Berkeley, USA: University of California Press.

Roychoudhury, N., Chandra, S., \& Singh, R. B. (2016). Pest status of gall making insect, Leptocybe invasa Fisher \& Lasalle, in nurseries and plantations of Eucalyptus in Madhya Pradesh. Indian Forester, 142(6), 601-606.

Senthilkumar, N., Murugesan, S., \& Thangapandian, K. (2013). Present status of eucalyptus gall insect, Leptocybe invasa (Fisher and La Salle) in Tamil Nadu. Current Science, 104(9), 1135-1136.

Shylesha, A. N. (2008). Classical biological control of eucalyptus gall wasp Leptocybe invasa Fisher \& La Salle. Final Project Report on the Management of Emerging Pests Eucalyptus. Recuperado de http://www.dcpulppaper.org/gifs/report63.pdf.

Steiner, A. (1961). A universal method for preparing 90 nutrient solutions of a certain desired composition. Plant Soil, 15, 134-154.

Stone, G. N. \& Schönrogge, K. (2003). The adaptive significance of insect gall morphology. Trends in Ecology and Evolution, 18(10), 512522. doi: $\underline{10.1016 / \text { S0169-5347(03)00247-7 }}$

Tang, C., Wang, X. J., Wan, F. H., Ren, S. X., \& Peng, Z. Q. (2008). The blue gum chalcid, Leptocybe invasa, invaded Hainan Province. Chinese Journal of Applied Entomology, 45, 967-971.
Thu, P. Q. Dell, B., \& Burgess, T. I. (2009). Susceptibility of 18 eucalypt species to the gall wasp Leptocybe invasa in the nursery and young plantations in Vietnam. Science Asia, 35, 113-117. doi: 10.2306/scienceasia1513-1874.2009.35.11

Tong, Y. G., Ding, X. X., Zhang, K. C., Yang, X., \& Huang, W. (2016). Effect of the Gall Wasp Leptocybe invasa on Hydraulic Architecture in Eucalyptus camaldulensis Plants. Frontiers in Plant Science, 7, 1-7. doi: $\underline{10.3389 / \text { fpls.2016.00130 }}$

Vanegas-Rico, J. M., Lomeli-Flores, J. R., Rodríguez-Leyva, E., JiménezQuiróz, E. \& Pujade-Villar, J. (2015). First record of eucalyptus gall wasp Leptocybe invasa (Hymenoptera: Eulophidae) in Mexico. Revista Mexicana de Biodiversidad, 86, 1095-1098. doi: 10.1016/j.rmb.2015.09.012

WilcKen, C. F. \& Filho, E. B. (2008). Vespa-Da-Galha do eucalipto (Leptocybe invasa) (Hymenoptera: Eulophidae), nova praga de florestas de eucalipto no Brasil. Instituto de Pesquisas e Estudios Florestais, pp. $11 . \quad$ Recuperado de http://www.ipef.br/protecao/alerta-leptocybe.invasa.pdf

Wiley, J. \& Skelly, P. (2008). A eucalyptus pest, Leptocybe invasa Fisher and La Salle (Hymenoptera: Eulophidae), genus and species new to Florida and North America. Florida Department of Agriculture and Consumer Services (US). Pest Alert. Recuperado de http://www.freshfromflorida.com/content/download/68487/1 614796/Pest Alert -

Leptocybe invasa, Blue Gum Chalcid.pdf.

Zhang, H. F., Kang, W. T., Chen, S. L., Tang, X. H., \& Lin, X. Q. (2012). Study on the relationship between eucalyptus clones and the damage degrees caused by Leptocybe invasa Fisher \& La Salle. Journal of Fujian College of Forestry, 32, 345-349.

Zheng, X. L., Li, J., Yang, Z. D., Xian, Z. H., Wei, J. G., Lei, C. L., \& Lu, W. (2014). A review of invasive biology, prevalence and management of Leptocybe invasa Fisher \& La Salle (Hymenoptera: Eulophidae: Tetrastichinae). African Entomology, 22(1), 68-79. doi: $10.4001 / 003.022 .0133$

Zheng, X. L.; Huang, Z. Y., Dong, D., Guo, C. H., Li, J., Yang, Z. D., Yang, X. H., \& Lu, W. (2016). Parasitoids of the eucalyptus gall wasp Leptocybe invasa (Hymenoptera: Eulophidae) in China. Parasite, 58, 1-7. doi: 10.1051/parasite/2016071

Manuscrito recibido el 3 de agosto de 2018

Aceptado el 15 de agosto de 2019

Publicado el 24 de abril de 2020 
Este documento se debe citar como:

Luna-Cruz, A., Vanegas-Rico, J. M., Rodríguez-Leyva, E., \& Lomeli-Flores, J. R. (2020). Susceptibilidad de Leptocybe invasa (Hymenoptera: Eulophidae) a insecticidas en invernadero. Madera y Bosques, 26(2), e2621835. doi: 10.21829/myb.2020.2621835

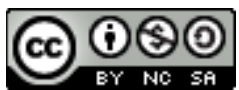

Madera y Bosques por Instituto de Ecología, A.C. se distribuye bajo una Licencia Creative Commons Atribución-NoComercialCompartirlgual 4.0 Internacional. 